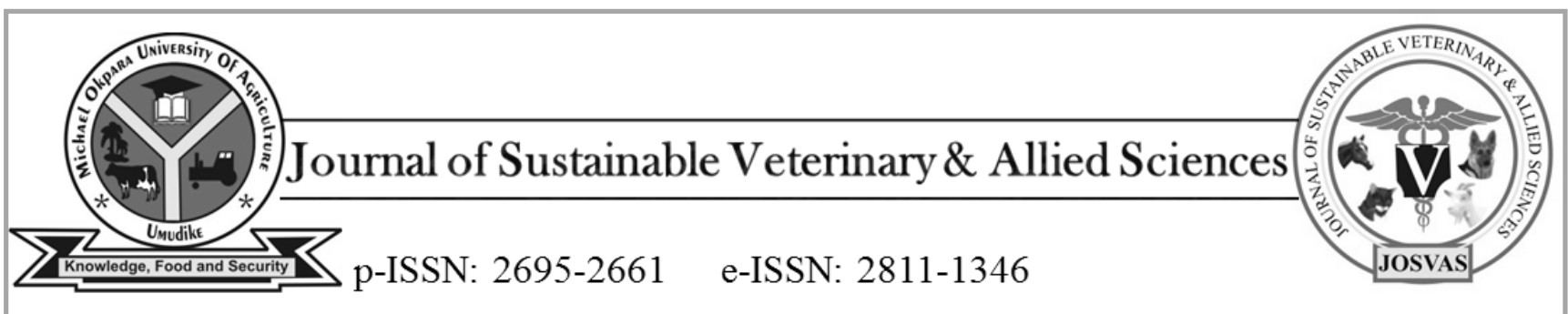

JoSVAS 2021, Vol 1, Issue 1: 96-99

C2021 College of Veterinary Medicine,

Michael Okpara University of Agriculture, Umudike, Nigeria

Case Report

\title{
Management of canine urolithiasis by cystotomy in a two-year old Lhasa Apso bitch
}

\author{
${ }^{1 *}$ Njoku, N.U., ${ }^{2}$ Ajayi, N.O., ${ }^{1}$ Kalu, N.R. \& ${ }^{1}$ Ukweni, C.P. \\ ${ }^{I}$ Department of Veterinary Surgery and Radiology, Michael Okpara University of Agriculture, Umudike, Abia State, ${ }^{2}$ Meck's \\ Veterinary Klinic Services, Port Harcourt, Rivers State, Nigeria.
}

*Corresponding author: njoku.njoku@ mouau.ed.ng, +2348063397918.

\begin{abstract}
Canine urolithiasis is a common urinary tract disease requiring a rapid definitive diagnosis for immediate surgical and/or nonsurgical therapy. The calculi may be microscopic or macroscopic, and when detected, the condition should be treated, either surgically or conservatively. This paper presents a surgical management of urolithiasis in a two-year old Lhasa apso bitch. The dog was haematuric for two weeks and had been on ceftriaxone and diclofenac for the period. Urolithiasis was confirmed following ultrasonography. The dog was pre-medicated with $0.02 \mathrm{mg} / \mathrm{kg}$ atropine sulphate and $2 \mathrm{mg} / \mathrm{kg}$ xylazine, while anaesthesia was induced and maintained with $10 \mathrm{mg} / \mathrm{kg}$ of $5 \%$ ketamine. Following aseptic patient preparation, the peritoneal cavity was accessed via a caudal ventral midline incision. The bladder was incised and the uroliths were evacuated via a cystotomy incision. Retrograde flushing of the urethra was performed to propel uroliths into the bladder for removal. The urinary bladder was closed with polyglycolic acid in Lambert suture pattern. The peritoneum and muscles were sutured using catgut in simple continuous suture. The skin was closed with silk using horizontal mattress suture pattern. The dog recovered uneventfully from the surgery and the wound healed after two weeks, at which time the skin suture was removed
\end{abstract}

Keywords: Bladder, calculi, cystotomy, urolith, urolithiasis.

\section{INTRODUCTION}

Urolithiasis is a common condition and often recurrent urogenital problem in dogs (Hoxha \& Rapti, 2018). Canine urolithiasis is a common cause of emergency urinary tract disease requiring a rapid definitive diagnosis for immediate surgical and/or medical therapy (Formsa \& Saini, 2019). Mechanisms involved in stone formation in dogs are not completely understood. However, some contributing factors include presence of inorganic protein core matrix, absence of natural crystal inhibitors and crystallization in urine solutes and other chemical substances that support crystal formation (Singh \& Sailo, 2013). Microscopic crystals associated with urolithiasis rarely cause clinical signs. The formation of macroscopic uroliths in the lower urinary tract irritates the mucosal surface resulting in dysuria, haematuria and stranguria. Other clinical signs include polakuria and polydipsia (Fossum et al., 2013; Hoxha \& Rapti, 2018).
Approach to diagnosis of urocystoliths may involve physical examination including abdominal palpation which may reveal a single large urolith or multiple uroliths by crepitation, rectal palpation and resistance at passing of urethral catheter (Herdrickson, 2007). Canine urolithiasis could be confirmed with imaging modalties. Survey radiographs are useful for detection radiopaque cystic calculi while radiolucent uroliths such as urate and cystine may require contrast radiography or ultrasonography to confirm their presence. Urinalysis of fresh warm urine, bacterial culture and sensitivity testing are critical parts of the evaluation and may help to determine the type of urolith present. Ultrasonography and cystoscopy may also be useful diagnostic modalities (Singh \& Sailo, 2013).

The urolith can be classified by quantitative techniques such as optical crystallography, polarized light microscopy, X-ray diffraction, Fourier-transform infrared spectroscopy, or scanning electron microscopy (Houston et al., 2004). Certain breeds of dogs are genetically predisposed to this condition 
with specific stone type formation. Oxalate stones are more predominant in Lhasa Apsos, urate stones in Dalmatians and English bulldogs, cystine stones in Newfoundlands, struvite and oxalate stones in miniature Schnauzers and Bichon frises (Houston et al., 2004; Houston \& Moore, 2009; Hoxha \& Rapti, 2018). Breeds frequently identified with struvite urolithiasis include the miniature Schnauzers, Shih Tzus, Lhasa Apso, Yorkshire Terriers and Pugs (Weichselbaum et al., 1998). Dog breeds at risk of the condition are the Pekingese and Cocker spaniel.

Treatment of urolithiasis can be surgical or nonsurgical. Conservative management involves dissolving of calculi with appropriate solutions/diet depending on the composition of the urolith (Siener, 2021). Although noninvasive techniques such as extracorporeal shock wave lithotripsy, ureteric stenting and laser lithotripsy are available in a few specialist referral centres, these are not widely available and are cost prohibitive for many pet owners (Adams et al., 2008; Berent, 2011). Surgical intervention involves incisional removal of calculi especially those that cannot be dissolved like calcium oxalate, urate, cystine and silicate stones from the urogenital tract depending on the size and where it is located. If left untreated, urolithiasis could lead to partial or complete obstruction of the urinary tract which can result in complications such as rupture of the urogenital tract, metabolic acidosis, hydronephrosis, hyperkalemia, peritonitis, cardiac arrhythmias and death in severe cases of obstruction (Appel et al., 2012; Singh \& Sailo, 2013; Tion et al., 2015).

The aim of this case presentation is to report the occurrence and surgical treatment of urolithiasis in a Lhasa Apso bitch, as well as to increase scholarly publications on the subject.

\section{CASE REPORT}

A two-year old spayed Lhasa apso bitch weighing $7.45 \mathrm{~kg}$ was presented with a compliant of haematuria and stranguria that was first noticed on $12^{\text {th }}$ December 2019. The bitch had a complete vaccination records, housed indoors and fed with both household and commercial diet. The dog had been on Ceftriaxone IV injections at $25 \mathrm{mg} / \mathrm{kg}$ twice daily, fluids and anti-inflammatory drugs for two weeks to treat a suspected urinary tract infection before it was referred to the clinic. The bitch was active with physiological parameters that were within the normal range. Blood samples were sent to the laboratory for haemoparasite screening, serum biochemistry and full blood count, while ultrasonography revealed hyperechoic areas within the urinary bladder which confirmed presence of cystic calculi. (Figure I A and B).

\section{SURGICAL INTERVENTION}

The dog was prepared aseptically prepared and positioned on dorsal recumbency. Atropine sulphate (Zhejiang Jinling Tianfeng Pharmaceutical Factory, China) at $0.03 \mathrm{mg} / \mathrm{kg}$ and xylazine hydrochloride (XYL - M2, VMD, Belgium) at 2 $\mathrm{mg} / \mathrm{kg}$ were administered intravenously as pre-anaesthetic medications, while ketamine hydrochloride injection (KETMIN, Laborate Pharmaceutical, India) was administered intramuscularly at $10 \mathrm{mg} / \mathrm{kg}$ for induction of anaesthesia. Ringer lactate intravenous fluid (BIOFLEX, Biomedical Limited, Nigeria) was administered at $10 \mathrm{mg} / \mathrm{kg} / \mathrm{hr}$ through the cephalic vein.

Retrograde urohydropropulsion was performed by passing a 6Fr urinary catheter (MEDIFLON, Global Medikit Limited, India) into the urethra and the calculi was propelled back into the bladder. The bladder was emptied of urine via the urinary catheter.

A $10 \mathrm{~cm}$ ventral midline incision, extending from the umbilicus to the pubis, was made on the linea alba into the peritoneum to expose the caudal abdominal viscera. The bladder was identified, exteriorized and stay sutures were placed on the apex and caudal part of the bladder to hold it in place.

A stab incision was made on the body of the bladder and incision was extended to about $2 \mathrm{~cm}$ with a pair of scissors to permit removal of the calculi from the bladder. Several calculi were removed (Fig II and III).and the bladder flushed with normal saline continuously

The cystotomy incision was closed with a double row Lambert suture pattern using size $2 / 0$ polyglycolic acid (Orion Sutures India Pvt Ltd, India). The peritoneum and muscles were also sutured using size $2 / 0$ chromic catgut (Orion Sutures India Pvt Ltd, India) in a simple continuous pattern, then the fascia was also apposed in like manner. Subcuticular sutures were first placed for skin closure using size 2/0 chromic catgut and then reinforced with $1 / 0$ silk (Orion Sutures India Pvt Ltd, India) applied in a horizontal mattress suture pattern.

Postoperatively, an Elizabethan collar was placed on the dog to avoid self-traumatization. Diclofenac injection at a dose of $0.3 \mathrm{mg} / \mathrm{kg}$ stat and oral Augmentin (Amoxycillin/Clavulanate, GSK, London) were administered at a dose of $20 / 5 \mathrm{mg} / \mathrm{kg}$ for 7 days. The dog vomited a day after surgery, and also passed a urolith in urine. Metoclopromide, $0.2 \mathrm{mg} / \mathrm{kg}$ (PETASIL, Hubei Tianyao Pharmaceutical, China) IV was given for 2 days. The urethral catheter was removed after 2 days while skin sutures were removed after 14 days. The owner was advised to provide liberal supply of water to the dog, feed with less salt, and stop all treats. The wound was examined daily for wound healing progress, and dressed appropriately. The bitch recovered uneventfully with restoration of normal urination after catheter was removed. 

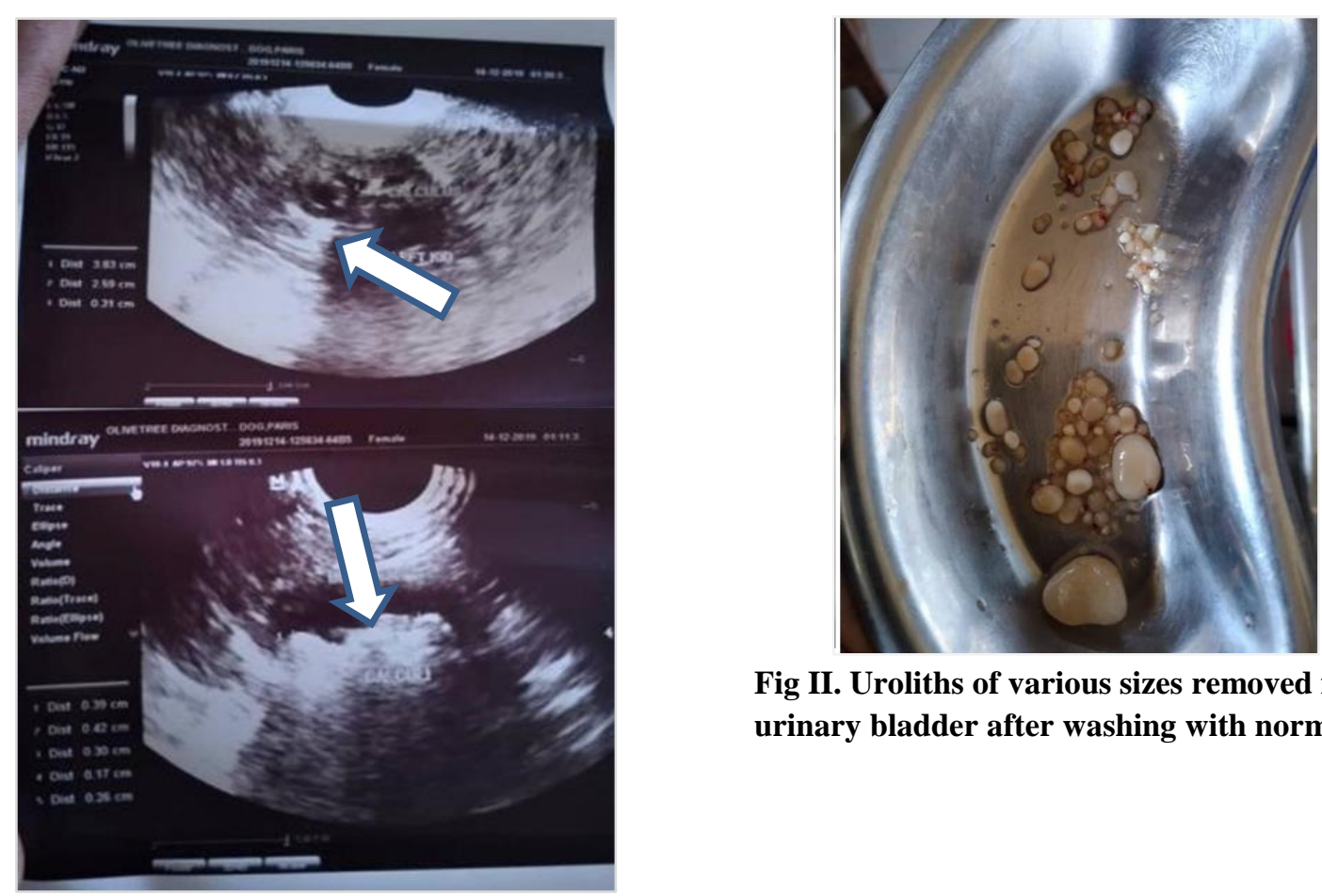

Fig II. Uroliths of various sizes removed from the urinary bladder after washing with normal saline

Figure I. Sonogram of the urinary bladder showing uroliths in white arrows.

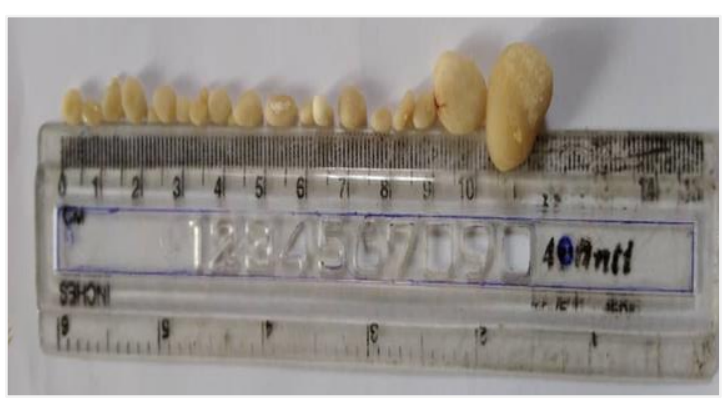

Fig III. Showing the actual sizes of the uroliths.

\section{DISCUSSION}

The incidence of urolithiasis in companion animals is reported to be higher than in humans due to their quadruped position (Syme, 2012). This position makes the ventral part of the bladder to be the most dependent side, resulting in the settling and accumulation of particulate matter such as stoneforming crystals. When these crystals remain in the urinary bladder without being passed out in urine, they aggregate and form large calculi (Syme, 2012). Stones of variable sizes observed in the present case, may have been formed by this mechanism.

Risk factors for urolithiasis in dogs reportedly include sex and breed (Mendoza-Lopez, 2019). According to Ling et al. (1998), female dogs were of higher risk of developing urinary calculi than males. There are also reports that the Lhasa Apso breed is among the dogs with the highest chances of developing urinary calculi (Ling et al., 1998; Houston et al., 2004). It is therefore not surprising that the reported case of urolithiasis occurred in a Lhasa apso bitch.

The type of calculi isolated from this case could not be confirmed because of the absence of equipment for such confirmation at the time and in the locality where the surgery was carried out. The type of urinary calculi cannot be classified based on the form, colour, size and shape alone (Osborne et al., 1999). Confirmation of calculi type is carried out by a combination of optical crystallography, polarized light microscopy, X-ray diffraction, Fourier transform infrared spectroscopy and scanning electron microscopy in a reference laboratory (Osborne et al., 1999).

Treatment for canine urolithiasis ranges from dietary change to medical and surgical interventions, depending on the type, amount and location of urolith. The surgical procedure adopted for the removal of the calculi was the traditional cystotomy as opposed to the more recent cystoscopic guided laser lithotripsy percutaneous cystolithotomy (Cléroux, 2018). The surgical technique used in this case is same as has been previously reported by Khan et al. (2013). Mild variations in surgical procedure have been reported in the literature. Maiti et al. (2020) reported that the bladder was sutured using polyglactin in Lambert pattern. Reddy (2017) also reported suturing the bladder using catgut in Cushing pattern. Provided the integrity of the anastomosis is not compromised, both 
methods do not result in worrisome post-operative complications. Thus, we used polyglactin in Lambert pattern in this case.

Certain complications such as urinary tract infection, uroabdomen, incomplete removal of calculi, reocurrence of urolithiasis and surgical site infection have been reported as the most common complications with urolithiasis managed by cystotomy (Appel et al., 2012). The incidence of these complications is linked to factors relating to the experience of the surgeon and the physical status of the patient (Fossum et al., 2013). In the case reported, incomplete removal of calculi, as evidenced by the passage of a calculi in urine the day after surgery, was the only complication noted in this case.

\section{CONCLUSION}

Full recovery of the dog following the procedure is an indication that the procedure is fast, safe and effective in removing all the uroliths. Medical treatment may take a longer time. It can therefore be concluded that cystotomy for urolith removal remains the procedure of choice in the treatment of confirmed cases of canine urolithiasis.

\section{CONFLICT OF INTEREST}

There are no conflicts of interest in this study.

\section{REFERENCES}

Adams, L.G., Berent, A.C., Moore, G.E., Bagley, D.H. (2008). Use of laser lithotripsy for fragmentation of uroliths in dogs: 73 cases (2005-2006). Journal American Veterinary Medical Association; 232:16801687.

Berent, A.C. (2011). Ureteral obstructions in dogs and cats: a review of traditional and new interventional diagnostic and therapeutic options. J Vet Emerg Crit Care, 21:86-103.

Cléroux, A. (2018). Minimally invasive management of uroliths in cats and dogs. Veterinary Clinics: Small Animal Practice., 48: 875-889.

Formsa, A. and Saini, N.S. (2019). Canine urolithiasis and concurrent urinary bladder abnormalities: symptoms, haematology, urinalysis and comparative radiographic and ultrasonographic diagnosis. Veterinary Medicine Open Journal, 4(1): 18-26.

Fossum, T.W., Dewey, C.W., Horn, C.V., Johnson, A.I., MacPhail, C.M., Radlinsky, M.G., Shulz, K. and Willard, M.D. (2013). Small Animal Surgery, $4^{\text {th }}$ Ed. Mosby Inc, Missouri.

Herdrickson, D.A. (2007). Techniques in Large Animal Surgery. Blackwell Publishers, Iowa.

Houston, D.M. and Moore, A.E.P. (2009) Canine and feline urolithiasis: Examination of over 50000 urolith submissions to the Canadian Veterinary Urolith Centre from 1998 to 2008. Canadian Veterinarian Journal, 50:1263-1268.

Houston, D.M., Moore, A.E.P., Favrin, M.G. and Hoff, B. (2004) Canine urolithiasis: A look at over 16000 urolith submissions to the Canadian Veterinary
Urolith Centre from February 1998 to April 2003. Canadian Veterinary Journal, 45 (3): 225 - 230.

Hoxha, Z and Rapti, D. (2018), Breed Predisposition Differences of Dogs with Urolithiasis in Tirana District. Anglisticum Journal (IJLLIS), 7(4); 1857 8187

Khan, I.U., Khan, M.A., Chaudhary, A.S., Ali, M.M., Imran, M., Ijaz, M., and Saleem, M.H. (2013). Evaluation of different suturing techniques for cystotomy closure in canines. The Journal of Animal \& Plant Sciences, 23(4): 981-985.

Ling, G.V., Thurmond, M.C., Choi, Y.K., et al. (2003). Changes in proportion of canine urinary calculi composed of calcium oxalate or struvite in specimens analyzed from 1981 through 2001. Journal of Veterinary Internal Medicine, 17(6): 817-823.

Maiti, S.K., Shivaraju, S., Amitha, B.S., Kalaiselvan, E., Mishra, M., Pipelu, W., Raguvaran, R and Khan, S (2020). Surgical management of obstructive urolithiasis in a dog. Archives of Veterinary and Animal Sciences, 2(2).

Reddy, K.J.M. (2017). Surgical management of canine Urolithiasis a report of 3 cases. The Pharma Innovation Journal, 6(12): 249-252.

Ross SJ, Osborne CA, Lulich JP, et al (1999). Canine and feline nephrolithiasis: epidemiology, detection, and management. Veterinary Clinics of North America: Small Animal Practice, 29(1):231-250.

Singh, K.B. and Sailo, S. (2013). Understanding epidemiology and etiologic factors of urolithiasis: an overview. Science Vission, 13(4): 169-174.

Wagner CA, Mohebbi N. (2010). Urinary pH and stone formation. Journal of Nephrology, 23 Suppl 16: S165-9. PMID: 21170875.

Weichselbaum RC, Feeney DA, Jessen CR, Osborne CA, Koehler L, Ulrich L. (1998). Evaluation of the morphologic characteristics and prevalence of canine urocystoliths from a regional urolith center. Amerdam Journal of Veterinary Research.; 59: 379-387.

Article history:

Received: June 26, 2021,

Revised: August 3, 2021, Accepted: August 25, 2021 